\title{
Quality of Life and Anxiety in Patients with First Diagnosed Non-Muscle Invasive Bladder Cancer Who Receive Adjuvant Bladder Therapy
}

\author{
Alexandros Vaioulis ${ }^{\mathrm{a}}$, Konstantinos Bonotis ${ }^{\mathrm{b}}$, Konstantinos Perivoliotis $^{\mathrm{c}}$, Yiannis Kiouvrekis ${ }^{\mathrm{d}, \mathrm{e}}$, \\ Stavros Gravas ${ }^{\mathrm{a}}$, Vasilios Tzortzis ${ }^{\mathrm{a}}$ and Anastasios Karatzas ${ }^{\mathrm{a}, *}$ \\ ${ }^{a}$ Department of Urology, Faculty of Medicine, School of Health Sciences, University of Thessaly, Larissa, Greece \\ ${ }^{\mathrm{b}}$ Department of Psychiatry, Faculty of Medicine, School of Health Sciences, University of Thessaly, Larissa, \\ Greece \\ ${ }^{\mathrm{c}}$ Department of Surgery, Faculty of Medicine, School of Health Sciences, University of Thessaly, Larissa, Greece \\ ${ }^{\mathrm{d}}$ Department of Public and Integrated Health, University of Thessaly, Karditsa, Greece \\ ${ }^{\mathrm{e}}$ Business School, University of Nicosia, Nicosia, Cyprus
}

Received 26 November 2020

Accepted 15 May 2021

Pre-press 4 June 2021

Published 31 August 2021

\begin{abstract}
.
BACKGROUND: Bladder cancer (BC) is one of the most common malignancies (4.5\% of all newly diagnosed cases worldwide). Most of the new BC cases are diagnosed as non-muscle invasive BC (NMIBC), needing continuous follow up after primary endoscopic therapy. Adjuvant bladder therapy with chemo- or immuno- agents, apart from the initial diagnosis, the strict surveillance program and the risk of recurrence, may have a major impact on the patients' physical and mental health.

OBJECTIVE: We evaluated anxiety and quality of life (QoL) in patients who underwent surgery for NMIBC and followed a bladder instillation programme.

METHODS: This is a prospective analysis of patients with histopathologically confirmed NMIBCs. Eligible were all adult patients with a single or multiple NMIBCs who underwent a transurethral tumor resection and followed a therapy with either BCG or Epirubicin instillations. The SF-36 questionnaire Physical and Mental health aspects were used for QoL assessment. Similarly, the STAI-Y was introduced for the state (STAI-Y1) and trait anxiety (STAI-Y2) evaluation.

RESULTS: 117 eligible patients were screened, with 108 entering finally the study; 9 patients were excluded due to disease recurrence. 17 patients (15.7\%) received Epirubicin (Ta-T1, Low Grade tumors), whereas 91 patients (84.3\%) received BCG (T1, High Grade). Regarding SF-36 Physical a 6 months decrease was followed by an improvement at 12 months $(p=0.008)$. Similarly, an increase of the SF-36 Mental health score was identified ( $p=0.03)$. In contrast to STAI-Y2 scores $(p=0.945)$, a
\end{abstract}

\footnotetext{
*Correspondence to: Anastasios D. Karatzas, MD, PhD, FEBU, FEAPU, Department of Urology, Faculty of Medicine, School of Health Sciences, University of Thessaly, Biopolis, 41110, Larissa,
}

Greece. Tel.: +00302413502988; Fax: +00302413500103; E-mail: karatzas@med.uth.gr. 
long-term reduction of the state anxiety was identified $(p=0.001)$. Preoperative SF-36 Physical was inversely correlated with age $(p=0.029)$, while absence of alcohol was associated with lower mental health $(p=0.003)$. Overall, patient characteristics, habits and the administered treatment did not affect the postoperative QoL and anxiety.

CONCLUSION: Patient QoL and anxiety improved during follow up. Still, further larger scale studies are required to support our findings.

Keywords: Non-muscle invasive bladder cancer (NMIBC), quality of life (QoL), anxiety, short form survey (SF-36)/State-Trait Anxiety Inventory (STAI), adjuvant bladder therapy

\section{INTRODUCTION}

Bladder cancer $(\mathrm{BC})$ is one of the most common malignancies, accounting for almost $4.5 \%$ of all newly diagnosed cases worldwide [1]. The annual incidence of BC is 180,000 new cases, whereas the mortality rate is estimated to be more than 55,000 patients per year. The gradually ageing population and the increased exposure to several risk factors are expected to further increment the burden of $\mathrm{BC}$ [2].

Most new $\mathrm{BC}$ cases are diagnosed as non-muscle invasive BC (NMIBC) [3]. Current approach for the NMIBC management is based on the transurethral resection of the tumor followed by a strict follow-up schedule that may include intravesical administration of chemotherapeutic or immune-modifying regimens. Even though 5-year overall survival of NMIBC is $96 \%$ [4], recurrence is quite common, with the 1st and the 5 th year rate being $33 \%$ and $50 \%$, respectively. As a result, patients are required to undergo successive cystoscopies and adjuvant treatments [5].

Initial diagnosis, the strict surveillance program and the risk of recurrence have a major impact on the patients' physical and mental health [6-8]. Furthermore, NMIBC intravesical treatment several adverse events, affecting patients' quality of life (QoL). The most common complaint of patients receiving bladder instillations for NMIBC is lower urinary tract symptoms (LUTS) [9]. Indeed, several studies attempted to evaluate factors of the detrimental effect of the NMIBC therapeutic approaches on the psychological and physical health, with methodological issues $[3,6,7,10]$. The application of generic and widely validated tools, such as SF-36 and STAI-Y, allows for the overall assessment of the QoL and anxiety, respectively [11]. However, current evidence regarding the perioperative QoL and anxiety patterns of NMIBC patients on the basis of SF-36 and STAI-Y are minimal [12].

In this study we tried to evaluate the QoL and the anxiety response in patients suffering from NMIBC who underwent surgery and they had adjuvant intravesical therapy with bladder instillations.

\section{METHODS}

\section{Inclusion criteria}

The present study is a prospective analysis of all patients who underwent surgery for NMIBC in our tertiary center, between 2018-2020 and were planned to receive adjuvant intravesical therapy (Fig. 1). As such, all adult patients (age: 18-80 years) with histopathologically confirmed NMIBC (pTis, pTa, pT1) entered our study. An institutional ethics approval was received (Approval number: 4212/2707-2018). All eligible patients provided a written informed consent. The study adhered to the Helsinki Declaration [13] and was reported on the basis of the STROBE guidelines [14].

\section{Exclusion criteria}

Patients with severe comorbidities (ASA $>$ III), muscular invasive bladder cancer, previous malignancy, recurrent bladder cancer, refusal to participate, and patients that were, either, not fluent Greek speakers, or who did not adhere to the follow up schedule were excluded.

\section{Study protocol}

All patients underwent endoscopic resection of the bladder tumor under spinal anesthesia. Three weeks postoperatively they initiated adjuvant intravesical therapy of either BCG or Epirubicin instillations on an outpatient basis, as described elsewhere [15].

Demographics (age, gender, educational level, marital status, residence and employment status) and habitual data (smoking, alcohol) were recorded for all eligible patients. The SF-36 questionnaire was introduced for the QoL assessment. This tool has been previously validated in the Greek population [16] and its efficacy in the overall QoL evaluation has been extensively confirmed in various settings [17]. All eight subscales of the questionnaire are sub-grouped in two major components, the Physical 


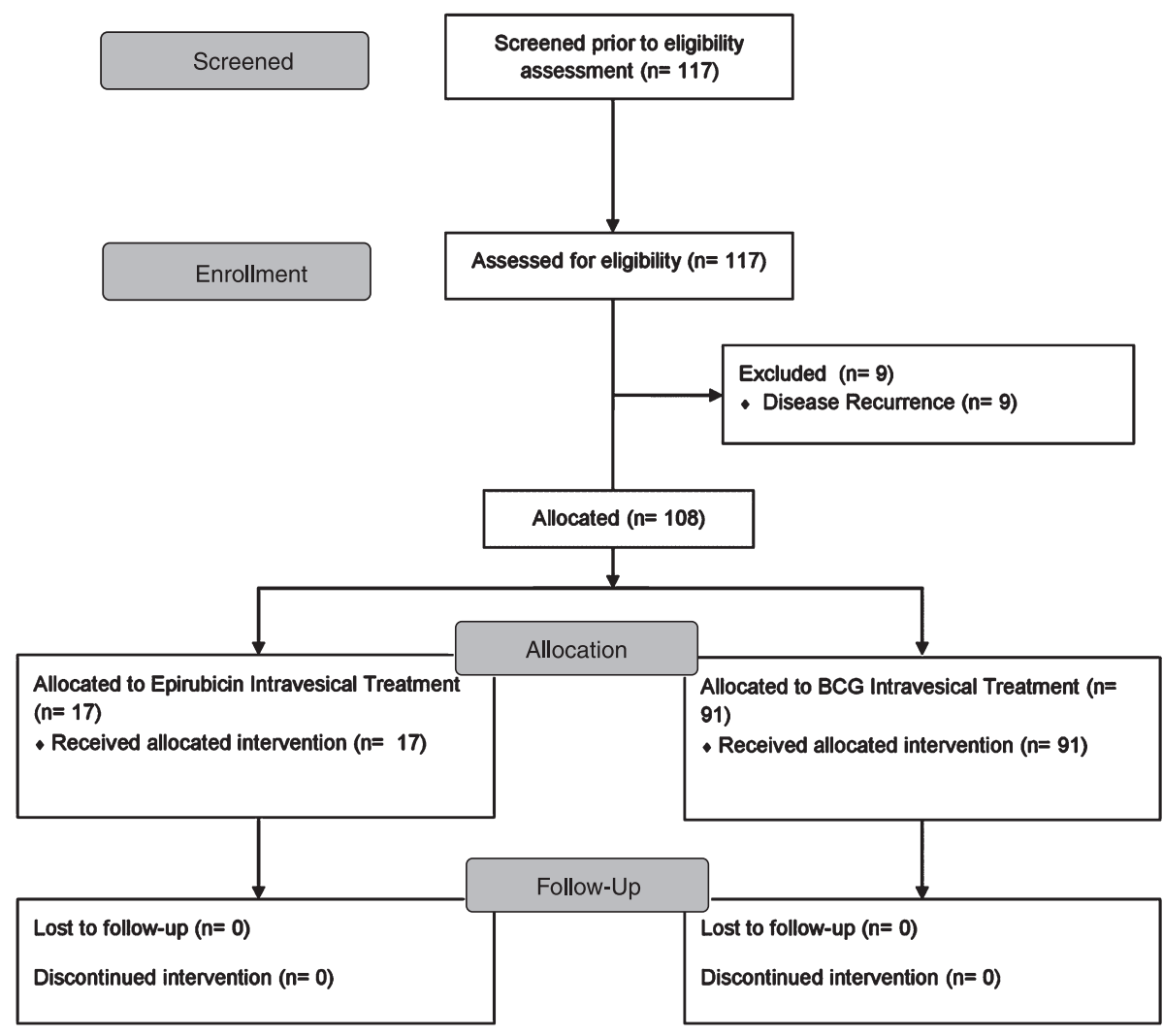

Fig. 1. Study flow-chart.

(Physical Functioning -PF, Role Physical-RP, Bodily Pain-BP and General Health-GH) and Mental (Vitality-VT, Mental Health-MH, Role EmotionalRE and Social Functioning-SF) health aspect. State anxiety echoes the psychological and physiological temporary way of reacting in a specific moment. On the other hand, the trait anxiety refers to a more stable pattern of behavior, reflecting someone's personality. Results range from 0 to 100, with the latter being the optimal outcome. Consistently, anxiety levels were estimated by the validated State-Trait Anxiety Inventory (STAI-Y) questionnaire [18]. The two subscales of this tool address the state (STAI-Y1) and trait anXiety (STAI-Y2) of the subject. Scores ranging from 29-39 were considered as low, whereas values greater that 60 were translated as increased anxiety. Patient evaluation was performed 2 weeks preoperatively and during the scheduled postoperative follow up (3, 6 and 12 months after surgery) examination. All data was secured in an encrypted electronic repository.

The primary endpoint of our study was the assessment of the SF-36 Physical health aspect variations during the follow up period. Secondary endpoints were the evaluation of the time fluctuations of SF36 Mental, STAI-Y1 and STAI-Y2. Moreover, an association analysis of patient characteristics with the preoperative and postoperative QoL and anxiety measurements was performed. Based on the reliability of the questionnaires, the minimum required number of sampling observations (power: $80 \%$, effect size: 0.3 , type I error: 5\%) was estimated at the level of 90 cases.

All data underwent a Shapiro-Wilk test for normality. Independent samples $t$ test was used for continuous variables. Given an incompetence to confirm normality, the evaluation of a possible relationship between distinct patient characteristics and the preoperative QoL and anxiety levels was based on the Mann-Whitney U and the Kruskal-Wallis H test. In order to examine the time-related variations of the questionnaire measurements, a repeated measures ANOVA model (RM-ANOVA) was introduced. Violation of the sphericity assumption was assessed by the Mauchly's test of sphericity. If a violation was confirmed, then a Greenhouse-Geisser correction was applied. Post hoc comparisons and interactions of 
time endpoints and patient characteristics were calculated. The effect of distinct characteristics on the measured variables was estimated by the F test. Cronbach's Alpha was, also, calculated for the evaluation of the internal consistency of the questionnaires in each time endpoint.

Categorical variables were provided as N (Percentage), whereas continuous data were reported as Mean (Standard Deviation). For all analyses, mean $\pm 2 \mathrm{SE}$ was displayed through all time endpoints. Statistical significance was considered at the level of $p<0.05$. All analyses were completed in SPSS version 23 software (SPSS Inc. Chicago, IL, USA).

\section{RESULTS}

117 eligible patients were initially screened, with 108 entering finally the study (97 males and 11 females); 9 patients were excluded due to disease recurrence. Patients' characteristics and habits are shown on Table 1. Most cases were never exposed to alcohol (35.2\%), in contrast to smoking (11.1\%). According to pathology, 17 patients (15.7\%) received epirubicin (Ta-T1, Low Grade tumors), whereas 91 patients $(84.3 \%)$ received BCG (T1, High Grade). Overall, $75 \%$ of patients had no therapy-related adverse event (Table 1 and Supplementary Material Tables), while dysuria and hematuria were reported in $19 \%$ and $8 \%$ respectively. All patients tolerated therapy well; there were no withdrawals due to therapy symptoms or technical issues (i.e, catheterization, instillation, etc).

The fluctuation of the SF-36 Physical over the progressive time endpoints is visualized in Fig. 2. Mean values at 3 months were comparable to the preoperative measurements. The 6 months decrease was followed by a significant improvement at 12 months. Overall, RM-ANOVA (Table 2) confirmed the presence of a significant time variation $(p=0.008)$.

Similarly, a significant $(p=0.03)$ increase of the SF-36 Mental health score (Fig. 3) was identified. As shown in Table 2, a higher 12-month mean value was estimated (72.2), compared to the respective preoperative assessment (64.2). In terms of anxiety, analysis confirmed a long-term decrease (Fig. 4) of the state anxiety (preoperative STAI-Y1:41.2, 12 months STAI-Y2:33, $p=0.001)$. In contrast to these, STAI-Y2 reports (Fig. 5) were consistent over the time endpoints $(p=0.945)$.

Preoperative SF-36 Physical scores were inversely $(p=0.029)$ correlated with age (Table 1 and Supplementary Material Figures). Absence of alcohol
Table 1

Patients' demographics

\begin{tabular}{|c|c|c|c|}
\hline & & $N$ & Percentage $(\%)$ \\
\hline \multirow[t]{2}{*}{ Gender } & Male & 97 & 89.8 \\
\hline & Female & 11 & 10.2 \\
\hline \multirow[t]{2}{*}{ Age } & $<66$ years & 40 & 37.0 \\
\hline & $66+$ years & 68 & 63.0 \\
\hline \multirow[t]{2}{*}{ Tumor Stage } & Ta-T1, Low Grade & 17 & 15.7 \\
\hline & T1, High Grade & 91 & 84.3 \\
\hline \multirow[t]{6}{*}{$\begin{array}{l}\text { Educational } \\
\text { Level }\end{array}$} & $\begin{array}{c}\text { No Primary } \\
\text { Education }\end{array}$ & 3 & 2.8 \\
\hline & Primary Education & 49 & 45.4 \\
\hline & Secondary Education & 26 & 24.1 \\
\hline & Higher Education & 26 & 24.1 \\
\hline & $\mathrm{MSc}$ & 4 & 3.7 \\
\hline & $\mathrm{PhD}$ & 0 & 0.0 \\
\hline \multirow[t]{5}{*}{ Marital Status } & Unmarried & 2 & 1.9 \\
\hline & Married & 92 & 85.2 \\
\hline & Divorced & 8 & 7.4 \\
\hline & Widowed & 4 & 3.7 \\
\hline & Cohabitation & 2 & 1.9 \\
\hline \multirow{3}{*}{ Residence } & Local Prefecture & 75 & 69.4 \\
\hline & Local Region & 27 & 25.0 \\
\hline & $\begin{array}{l}\text { Outside Local } \\
\text { Region }\end{array}$ & 6 & 5.6 \\
\hline \multirow{4}{*}{$\begin{array}{l}\text { Employment } \\
\text { Status }\end{array}$} & Employed & 20 & 18.5 \\
\hline & Retired & 83 & 76.9 \\
\hline & Unemployed & 4 & 3.7 \\
\hline & Household & 1 & 0.9 \\
\hline \multirow[t]{3}{*}{ Smoking } & No & 12 & 11.1 \\
\hline & Ex-smoker & 60 & 55.6 \\
\hline & Yes & 36 & 33.3 \\
\hline \multirow[t]{3}{*}{ Alcohol } & No & 38 & 35.2 \\
\hline & Social consumption & 56 & 51.9 \\
\hline & Daily & 14 & 13.0 \\
\hline \multirow[t]{2}{*}{ Treatment } & $\mathrm{BCG}$ & 91 & 84.3 \\
\hline & Epirubicin & 17 & 15.7 \\
\hline \multirow[t]{3}{*}{ Adverse Events } & None & 81 & 75 \\
\hline & Dysuria & 19 & 19 \\
\hline & $\begin{array}{r}\text { Microscopic } \\
\text { Hematuria }\end{array}$ & 8 & 8 \\
\hline
\end{tabular}

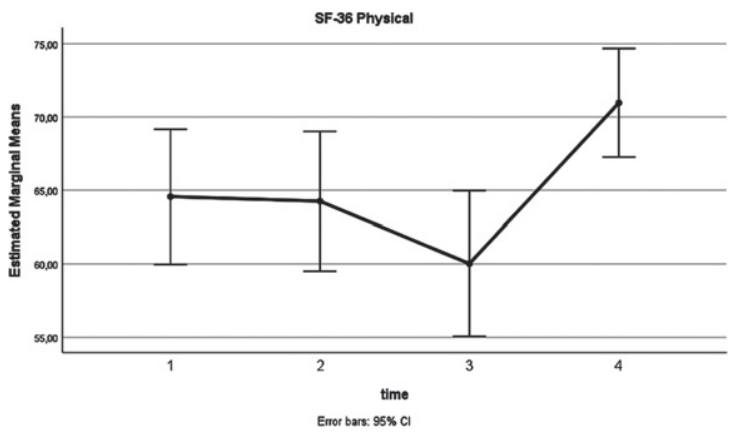

Fig. 2. SF-36 Physical (x-axis: 1: preoperatively, 2: at 3 months postoperatively, 3: at 6 months postoperatively, 4: at 12 months postoperatively). 
Table 2

Basic questionnaire results

\begin{tabular}{|c|c|c|c|c|c|}
\hline Subscale & Time & Mean & $\begin{array}{c}\text { Standard } \\
\text { Deviation }\end{array}$ & $\begin{array}{c}\text { Mauchly's } \\
\text { Sphericity } P\end{array}$ & $\begin{array}{c}\text { Time } \\
\text { Variation } P \\
\end{array}$ \\
\hline \multirow{4}{*}{ SF-36 Physical } & 2 weeks preoperatively & 64.6 & 24.1 & 0.011 & $\mathbf{0 . 0 0 8}^{*}$ \\
\hline & 3 months postoperatively & 64.3 & 24.9 & & \\
\hline & 6 months postoperatively & 60.0 & 25.9 & & \\
\hline & 12 months postoperatively & 71.0 & 19.4 & & \\
\hline \multirow[t]{4}{*}{ SF-36 Mental } & 2 weeks preoperatively & 64.2 & 24.4 & 0.011 & $\mathbf{0 . 0 3} *$ \\
\hline & 3 months postoperatively & 61.1 & 26.2 & & \\
\hline & 6 months postoperatively & 64.1 & 22.7 & & \\
\hline & 12 months postoperatively & 72.2 & 18.3 & & \\
\hline \multirow[t]{4}{*}{ STAI-Y1 } & 2 weeks preoperatively & 41.2 & 11.1 & 0.023 & $0.001 *$ \\
\hline & 3 months postoperatively & 42.1 & 14.7 & & \\
\hline & 6 months postoperatively & 40.7 & 13.6 & & \\
\hline & 12 months postoperatively & 33.0 & 9.3 & & \\
\hline \multirow[t]{4}{*}{ STAI-Y2 } & 2 weeks preoperatively & 44.0 & 9.3 & 0.362 & 0.945 \\
\hline & 3 months postoperatively & 44.0 & 11.3 & & \\
\hline & 6 months postoperatively & 44.6 & 12.1 & & \\
\hline & 12 months postoperatively & 43.8 & 11.8 & & \\
\hline
\end{tabular}

*Greenhouse-Geisser correction applied STAI score: 29-39: low anxiety 40-59: moderate anxiety 60-80: high anxiety SF-36 score: 0: minimum value 100: maximum value.

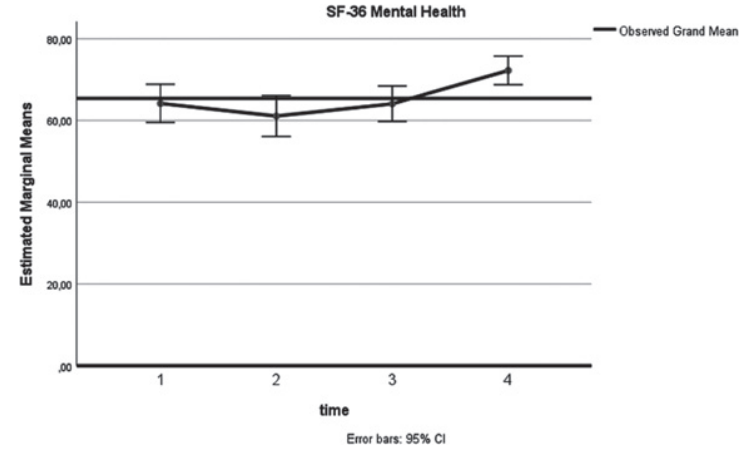

Fig. 3. SF-36 Mental (x-axis: 1: preoperatively, 2: at 3 months postoperatively, 3: at 6 months postoperatively, 4: at 12 months postoperatively).

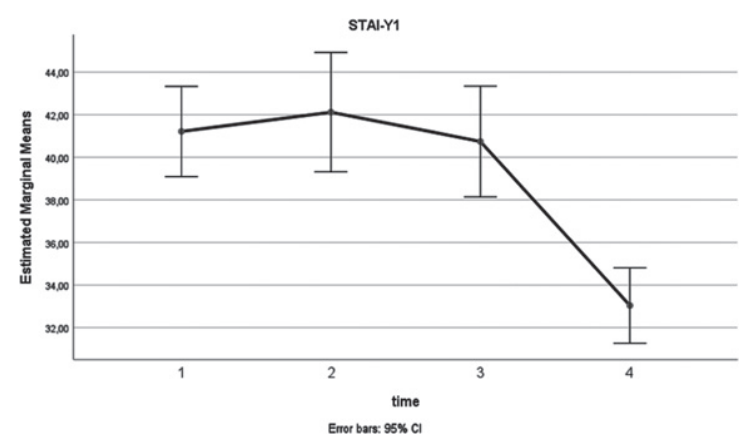

Fig. 4. STAI-Y1 (X-axis: 1: preoperatively, 2: at 3 months postoperatively, 3: at 6 months postoperatively, 4: at 12 months postoperatively).

consumption was associated with significantly lower values in mental health (SF-36 Mental $p=0.003$ ). Gender, educational level and smoking did not affect

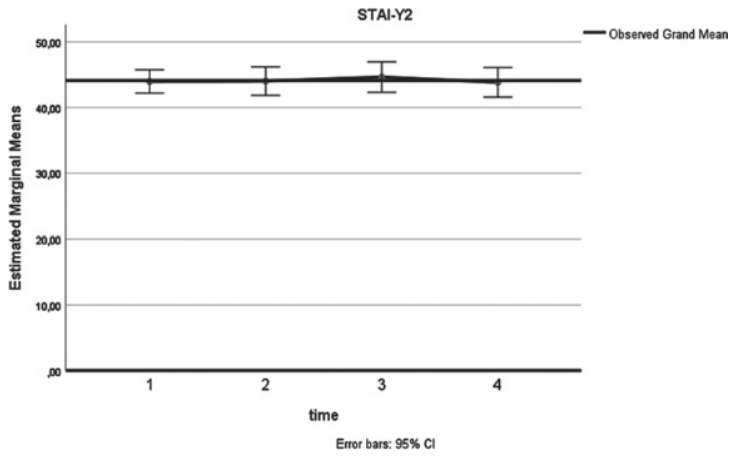

Fig. 5. STAI-Y (X-axis: 1: preoperatively, 2: at 3 months postoperatively, 3: at 6 months postoperatively, 4: at 12 months postoperatively).

the preoperative QoL and anxiety levels, while tumor stage and residence status correlated with the preoperative STAI-Y1 scores (Table 3 and Supplementary Material Figures).

A significant interaction of age $(p=0.011)$ and alcohol consumption $(p=0.015)$ with time in the Physical aspect of the SF-36 was documented (Supplementary Material Figures). However, there was no significant effect $(p=0.605$ and $p=0.89)$ of these variables on the mean tool measurements. Moreover, a significantly different pattern of SF-36 Mental $(p=0.02)$, STAI-Y1 $(p=0.011)$ and STAI-Y2 $(p=0.003)$ scores, over the successive time endpoints was identified in the various alcohol consumption subgroups. A significant effect of alcohol was not confirmed in any tool. Overall, patient characteristics, habits, the administered treatment and the adverse 
Table 3

The effect of patient characteristics on quality of life and anxiety measurements

\begin{tabular}{|c|c|c|}
\hline Subscale & Factor & $P$ \\
\hline \multirow[t]{8}{*}{ SF-36 Physical } & Gender & 0.499 \\
\hline & Age & 0.029 \\
\hline & Tumor Stage & 0.478 \\
\hline & Educational Level & 0.188 \\
\hline & Marital Status & 0.475 \\
\hline & Residence & 0.123 \\
\hline & Smoking & 0.862 \\
\hline & Alcohol & 0.325 \\
\hline \multirow[t]{8}{*}{ SF-36 Mental } & Gender & 0.733 \\
\hline & Age & 0.483 \\
\hline & Tumor Stage & 0.109 \\
\hline & Educational Level & 0.386 \\
\hline & Marital Status & 0.413 \\
\hline & Residence & 0.878 \\
\hline & Smoking & 0.424 \\
\hline & Alcohol & 0.003 \\
\hline \multirow[t]{8}{*}{ STAI-Y1 } & Gender & 0.764 \\
\hline & Age & 0.597 \\
\hline & Tumor Stage & 0.04 \\
\hline & Educational Level & 0.299 \\
\hline & Marital Status & 0.361 \\
\hline & Residence & 0.040 \\
\hline & Smoking & 0.313 \\
\hline & Alcohol & 0.109 \\
\hline \multirow[t]{8}{*}{ STAI-Y2 } & Gender & 0.452 \\
\hline & Age & 0.386 \\
\hline & Tumor Stage & 0.46 \\
\hline & Educational Level & 0.495 \\
\hline & Marital Status & 0.170 \\
\hline & Residence & 0.213 \\
\hline & Smoking & 0.575 \\
\hline & Alcohol & 0.102 \\
\hline
\end{tabular}

events did not affect the postoperative QoL and anxiety (Supplementary Material Figures).

Based on the sample size, the power of the present study was estimated at the level of $87.1 \%$. Post-hoc analysis of the questionnaires' internal consistency revealed an adequate level of validity (Cronbach Alpha: 72.4\%-93.7\%) (Table 4). Exceptions were the 6-months SF-36 Mental and the 12-months STAI-Y1 measurements ( $6 \%$ and $35 \%$ respectively).

\section{DISCUSSION}

\section{Summary of evidence}

QoL corresponds to the gratification level of an individual's physical, mental, and social needs and therefore is accomplished through the interaction of various components. The inherent difficulty in differentiating between the subjective and objective components renders QoL assessment a challenging task [19]. Taking into consideration the variability of the available techniques, valid QoL evaluation is based on the suitability of the applied methods. Although the design of most of these tools address samples with heterogeneous base characteristics, the QoL assessment in specific patient categories require the introduction of specified questionnaires. Overall, QoL assessment tools are assigned in two subgroups. General indicators study generic aspects of physical and psycho-social functionality, whether specific assessment tools emphasize on the individual's perception regarding health and HRQoL [20].

NMIBC has a significant impact on a patient's QoL. The initial cancer diagnosis, the frequent cystoscopies and the adverse events related to the intravesical therapy affect the physical function, the mental health, the reported fatigue and the role function [6]. The detrimental effect of a BC diagnosis on QoL was reported by Singer et al. [21]. Even though no differences between non-muscle invasive and muscle-invasive bladder cancer patients were found, both groups had decreased physical and emotional functioning compared to the general population. Interestingly, QoL increased with time in all patient groups $(p<0.001)$.

Similarly, Fung et al. confirmed a significant postdiagnosis reduction of the physical and mental scores between the prediagnosis and post-diagnosis groups $(-2.7,95 \% \mathrm{CI}-3.8,-1.7$ vs $-1.4,95 \% \mathrm{CI}-2.6,-0.3)$. In patients with non-muscle invasive bladder cancer the physical and mental score differences were -1.9 $(p<0.01)$ and $-1.4(p=0.01)$, respectively. In those with muscle invasive bladder cancer there was a statistically and clinically significant difference in the physical but not the mental score $(-5.3, p<0.01$ vs $-2.7, p=0.07)$. The effect on the physical domain had a duration of 10 years [22].

In a prospective analysis by Schmidt et al., it was estimated that the impact of NMIBC on mental health was detectable during the 6-month follow-up period [23]. Mental health was significantly worse than SF-36 scores at diagnosis (mean of 49.7 vs. 53.3, 95\% CI: 52.5-54.2). Urinary domain improved significantly from diagnosis $(85.2,95 \%$ CI: 82.9-87.4) to 12-month evaluation (90.2, 95\% CI: 87.7-92.8), whereas sexual domain decreased from 56.4 (95\% CI: 52.8-59.9) to 53.7 (95\% CI: 50.0-57.4). However, in case of multiple TURBs, it was found that the initial impairment on mental health, gradually ameliorated. In terms of emotional role and physical and social functioning, a reduction on the estimated scores was confirmed at the 2nd or 3rd reoperation [17].

In our study, SF-36 physical health scores reached nadir at 6 months postoperatively, whereas the 
Table 4

Internal consistency of the questionnaires' subscales using Cronbach Alpha

\begin{tabular}{|c|c|c|c|c|}
\hline Subscale & Factor & $\begin{array}{l}\text { Mauchly's } \\
\text { Sphericity } P\end{array}$ & $\begin{array}{l}\text { Interaction } \\
\text { between Factor } \\
\text { and Time } P\end{array}$ & $\begin{array}{c}\text { Overall } \\
\text { Effect of } \\
\text { Factor } P\end{array}$ \\
\hline \multirow[t]{8}{*}{ SF-36 Physical } & Treatment & 0.013 & $0.216^{*}$ & 0.428 \\
\hline & Gender & 0.013 & $0.558^{*}$ & 0.776 \\
\hline & Age & 0.002 & $0.011^{*}$ & 0.605 \\
\hline & Educational Level & 0.012 & $0.222^{*}$ & 0.863 \\
\hline & Marital Status & 0.017 & $0.754^{*}$ & 0.085 \\
\hline & Residence & 0.011 & $0.479^{*}$ & 0.529 \\
\hline & Smoking & 0.003 & $0.108^{*}$ & 0.419 \\
\hline & Alcohol & 0.012 & $0.015^{*}$ & 0.89 \\
\hline \multirow[t]{8}{*}{ SF-36 Mental } & Treatment & 0.023 & $0.06^{*}$ & 0.83 \\
\hline & Gender & 0.009 & $0.265^{*}$ & 0.379 \\
\hline & Age & 0.012 & $0.241^{*}$ & 0.822 \\
\hline & Educational Level & 0.022 & $0.237^{*}$ & 0.785 \\
\hline & Marital Status & 0.021 & $0.502^{*}$ & 0.203 \\
\hline & Residence & 0.006 & $0.291^{*}$ & 0.455 \\
\hline & Smoking & 0.007 & $0.816^{*}$ & 0.641 \\
\hline & Alcohol & 0.006 & $0.02^{*}$ & 0.156 \\
\hline \multirow[t]{8}{*}{ STAI-Y1 } & Treatment & 0.051 & 0.059 & 0.253 \\
\hline & Gender & 0.016 & $0.4^{*}$ & 0.872 \\
\hline & Age & 0.025 & $0.84^{*}$ & 0.307 \\
\hline & Educational Level & 0.043 & $0.811^{*}$ & 0.60 \\
\hline & Marital Status & 0.025 & $0.758^{*}$ & 0.962 \\
\hline & Residence & 0.006 & $0.117^{*}$ & 0.672 \\
\hline & Smoking & 0.027 & $0.802^{*}$ & 0.707 \\
\hline & Alcohol & 0.017 & 0.011* & 0.071 \\
\hline \multirow{8}{*}{ STAI-Y2 } & Treatment & 0.305 & 0.157 & 0.798 \\
\hline & Gender & 0.341 & 0.71 & 0.809 \\
\hline & Age & 0.398 & 0.446 & 0.22 \\
\hline & Educational Level & 0.398 & 0.229 & 0.763 \\
\hline & Marital Status & 0.301 & 0.799 & 0.596 \\
\hline & Residence & 0.291 & 0.377 & 0.346 \\
\hline & Smoking & 0.516 & 0.202 & 0.828 \\
\hline & Alcohol & 0.179 & 0.003 & 0.198 \\
\hline
\end{tabular}

*Greenhouse-Geisser correction applied.

Table 5

Internal consistency of the questionnaires' subscales using Cronbach Alpha

\begin{tabular}{lcc}
\hline Subscale & Time & $\begin{array}{c}\text { Cronbach } \\
\text { Alpha (\%) }\end{array}$ \\
\hline SF-36 Physical & 2 weeks preoperatively & 72.4 \\
& 3 months postoperatively & 78.8 \\
& 6 months postoperatively & 80.2 \\
SF-36 Mental & 12 months postoperatively & 81.7 \\
& 2 weeks preoperatively & 83.1 \\
& 3 months postoperatively & 86.4 \\
6TAI-Y1 & 12 months postoperatively & 6.0 \\
& 2 weeks preoperatively & 78.7 \\
& 3 months postoperatively & 88.6 \\
& 6 months postoperatively & 93.7 \\
STAI-Y2 & 12 months postoperatively & 35.8 \\
& 2 weeks preoperatively & 80.1 \\
& 3 months postoperatively & 86.6 \\
& 6 months postoperatively & 87.0 \\
& 12 months postoperatively & 84.8 \\
\hline
\end{tabular}

mental component was minimized at 3 months. A gradual improvement that reached higher values compared to the preoperative scores over the remaining follow-up period was identified in both tools. These findings are in contrast with the existing literature. In the BOXIT trial, patients with no NMIBC progression or recurrence reached a minimal EQ-5D score at 2 months postoperatively, with no further improvement [24]. Cases of grade III recurrence and progression were associated with statistically significant HRQoL decrease $(-0.08 ; 95 \%$ confidence interval $[\mathrm{CI}],-0.13$ to -0.03 ; and $-0.10 ; 95 \% \mathrm{CI},-0.17$ to -0.03 , respectively).

Our data, also, suggested a significant association of age and alcohol with the Physical and Mental health, respectively. Similarly, in a study of 1160 $\mathrm{BCs}$, Yu et al., reported an improved physical component in young and healthy, single males, that did not smoke and were diagnosed with a NMIBC. Moreover, gender, age, tumor stage and comorbidity status, 
also, affected preoperative mental health. The biggest effect was found in the Physical Functioning domain, where a 10-year increase in age would decrease the score by 4 points. [3]. Although various patient characteristics interacted with time QoL measurements, none of them was an independent QoL affecting factor. Evidence from recent studies, though, suggest that postoperative HRQoL is significantly associated with modifiable behaviors such as physical activity, dietary habits and smoking. Sufficient physical activity, healthy diet, and non-smoking were significantly associated with HRQoL $(p<0.001)$ [7].

Likewise, anxiety levels are an important determinant of the treatment efficiency. Anxiety is defined as the unpleasant sense of fear in an unknown and ill-defined threat, followed by a variety of emotional and hemodynamic manifestations. Assessment of anxiety is based on the evaluation of both state and trait anxiety levels [18]. Preoperative anxiety is a well-documented entity with almost $80 \%$ incidence [25]. The physical and emotional distress symptoms have a wide severity range and can present several weeks preoperatively [26]. Furthermore, increased perioperative anxiety is related to suboptimal patient recovery, impaired immunologic function and increased morbidity and mortality. Despite initial diagnosis, socioeconomic factors, such as educational and marital status were predictive factors of preoperative anxiety. Preoperative distress, combined with postoperative pain and smoking are considered as independent postoperative anxiety risk predictors [27].

Regardless of a curative or a palliative approach, anxiety and depression rates in cancer patients are estimated at the level of $10 \%$ and $20 \%$, respectively [28]. The significant association of $\mathrm{BC}$ diagnosis and management with anxiety is supported by the reported depression and anxiety rate. In a recent meta-analysis, pooled postoperative depression and anxiety rates ranged at the levels of $4.7-78 \%$ and $12.5-71.3 \%$, respectively [29]. The long term duration of NMIBC treatment results to the development of post-traumatic stress disorder (PTSD) syndrome [30]. In this cohort, it was confirmed that uncertainty and PTSD symptoms were inversely related to QoL as it was quantified by the EORTC QLQ-30 and QLQ-NMIBC24. Although post-resection anxiety and depression status lessens gradually, tumor and operative characteristics, catheterization time and morbidity rates significantly affect overall distress levels. Our analyses showed a significant decrease of state anxiety, in contrast to the trait component, which remained stable. This could be attributed to the fact that since the patients were familiar to the follow-up process, they could more easily manage their reactions. These reactions though could not possibly alter their character and personal behavior in general. Both preoperative and postoperative STAI-Y scores were not associated to the study characteristics.

Another interesting fact in our study is that QoL and anxiety scores showed no statistically difference in terms of the preferred adjuvant therapy. Major concern for both epirubicin and BCG patients seemed to be the procedure itself (i.e, waiting, urethral catheterization, instillation, LUTS, etc) and not the recurrence or disease progress. There were no withdrawals due to LUTS or technical issues. Since they tolerated therapy well, all patients improved their scores over time.

Study limitations are the prospective methodology and non-randomization. Furthermore, there was no risk stratification of NMIBC patients (grade and staging) and no pre-cancer data on anxiety was collected. Additionally, no data on impact of financial/logistical constraints on QoL and anxiety were collected, even though this was not our aim. Although the statistical power of our findings was adequate, our sample size is relatively small. Finally, a longer follow up postoperatively period is needed to draw further conclusions regarding anxiety and QoL in patients with NMIBC.

In conclusion, an improvement in the Physical and Mental health aspect was identified during the follow-up period in patients receiving adjuvant bladder therapy for NMIBC. Furthermore, in contrast to Trait anxiety, State stress levels displayed a gradual declining trend. Preoperative Physical health was inversely correlated with age, while absence of alcohol was associated with lower Mental health. Overall, patient characteristics, habits and the administered treatment did not affect the postoperative QoL and anxiety. Due to several study limitations, larger scale studies with an extended follow up period are required for safe conclusions.

\section{ACKNOWLEDGMENTS}

The authors have no acknowledgments.

\section{FUNDING}

The authors report no funding. 


\section{AUTHOR CONTRIBUTIONS}

Alexandros Vaioulis: performance of work; writing the article. Konstantinos Bonotis: conception; performance of work. Konstantinos Perivoliotis: performance of work; interpretation or analysis of data. Yiannis Kiouvrekis: performance of work; interpretation or analysis of data; Stavros Gravas: performance of work. Vasilios Tzortzis: performance of work. Anastasios Karatzas: conception; performance of work; interpretation or analysis.

\section{CONFLICT OF INTEREST}

Alexandros Vaioulis has no conflict of interest to report. Konstantinos Bonotis has no conflict of interest to report. Konstantinos Perivoliotis has no conflict of interest to report. Yiannis Kiouvrekis has no conflict of interest to report. Stavros Gravas has no conflict of interest to report. Vasilios Tzortzis has no conflict of interest to report. Anastasios Karatzas has no conflict of interest to report.

\section{SUPPLEMENTARY MATERIAL}

The supplementary material is available in the electronic version of this article: https://dx.doi.org/ 10.3233/BLC-201524.

\section{REFERENCES}

[1] Bray F, Ferlay J, Soerjomataram I, Siegel RL, Torre LA, Jemal A. Global cancer statistics 2018: GLOBOCAN estimates of incidence and mortality worldwide for 36 cancers in 185 countries. CA Cancer J Clin. 2018;68(6):394-424.

[2] Richters A, Aben KKH, Kiemeney L. The global burden of urinary bladder cancer: an update. World J Urol. 2020;38(8):1895-904.

[3] Yu EY, Nekeman D, Billingham LJ, James ND, Cheng KK, Bryan RT, et al. Health-related quality of life around the time of diagnosis in patients with bladder cancer. BJU Int. 2019;124(6):984-91.

[4] Steinberg RL, Thomas LJ, Brooks N, Mott SL, Vitale A, Crump T, et al. Multi-Institution Evaluation of Sequential Gemcitabine and Docetaxel as Rescue Therapy for Nonmuscle Invasive Bladder Cancer. J Urol. 2020;203(5):902-9.

[5] Babjuk M, Burger M, Comperat EM, Gontero P, Mostafid $\mathrm{AH}$, Palou J, et al. European Association of Urology Guidelines on Non-muscle-invasive Bladder Cancer (TaT1 and Carcinoma In Situ) - 2019 Update. Eur Urol. 2019;76(5):639-57.

[6] Jung A, Nielsen ME, Crandell JL, Palmer MH, Smith $\mathrm{SK}$, Bryant AL, et al. Health-related quality of life among non-muscle-invasive bladder cancer survivors: a population-based study. BJU Int. 2020;125(1):38-48.

[7] Chung J, Kulkarni GS, Bender J, Breau RH, Guttman D, Maganti M, et al. Modifiable lifestyle behaviours impact the health-related quality of life of bladder cancer survivors. BJU Int. 2020;125(6):836-42.

[8] Smith AB, Jaeger B, Pinheiro LC, Edwards LJ, Tan HJ, Nielsen ME, et al. Impact of bladder cancer on health-related quality of life. BJU Int. 2018;121(4):549-57.

[9] Mohamed NE, Gilbert F, Lee CT, Sfakianos J, Knauer C, Mehrazin R, et al. Pursuing Quality in the Application of Bladder Cancer Quality of Life Research. Bladder Cancer. 2016;2(2):139-49.

[10] Wettstein MS, Naimark D, Hermanns T, Herrera-Caceres JO, Ahmad A, Jewett MAS, et al. Required efficacy for novel therapies in BCG-unresponsive non-muscle invasive bladder cancer: Do current recommendations really reflect clinically meaningful outcomes? Cancer Med. 2020;9(10):3287-96.

[11] Chen TH, Li L, Kochen MM. A systematic review: how to choose appropriate health-related quality of life (HRQOL) measures in routine general practice? J Zhejiang Univ Sci B. 2005;6(9):936-40.

[12] Colombo R, Rocchini L, Suardi N, Benigni F, Colciago G, Bettiga A, et al. Neoadjuvant short-term intensive intravesical mitomycin $\mathrm{C}$ regimen compared with weekly schedule for low-grade recurrent non-muscle-invasive bladder cancer: preliminary results of a randomised phase 2 study. Eur Urol. 2012;62(5):797-802.

[13] Morris K. Revising the Declaration of Helsinki. Lancet. 2013;381(9881):1889-90.

[14] von Elm E, Altman DG, Egger M, Pocock SJ, Gotzsche PC, Vandenbroucke JP, et al. The Strengthening the Reporting of Observational Studies in Epidemiology (STROBE) statement: guidelines for reporting observational studies. J Clin Epidemiol. 2008;61(4):344-9.

[15] Mitrakas LP, Zachos IV, Tzortzis VP, Gravas SA, Rouka EC, Dimitropoulos KI, et al. Previous Bladder Cancer History in Patients with High-Risk, Non-muscle-invasive Bladder Cancer Correlates with Recurrence and Progression: Implications of Natural History. Cancer Res Treat. 2015;47(3):495-500.

[16] Pappa E, Kontodimopoulos N, Niakas D. Validating and norming of the Greek SF-36 Health Survey. Qual Life Res. 2005;14(5):1433-8.

[17] Yoshimura K, Utsunomiya N, Ichioka K, Matsui Y, Terai A, Arai Y. Impact of superficial bladder cancer and transurethral resection on general health-related quality of life: an SF-36 survey. Urology. 2005;65(2):290-4.

[18] Fountoulakis KN, Papadopoulou M, Kleanthous S, Papadopoulou A, Bizeli V, Nimatoudis I, et al. Reliability and psychometric properties of the Greek translation of the State-Trait Anxiety Inventory form Y: preliminary data. Ann Gen Psychiatry. 2006;5:2.

[19] Moons P, Budts W, De Geest S. Critique on the conceptualisation of quality of life: a review and evaluation of different conceptual approaches. Int J Nurs Stud. 2006;43(7): 891-901.

[20] Cunillera O, Tresserras R, Rajmil L, Vilagut G, Brugulat $\mathrm{P}$, Herdman $\mathrm{M}$, et al. Discriminative capacity of the EQ-5D, SF-6D, and SF-12 as measures of health status in population health survey. Qual Life Res. 2010;19(6): 853-64.

[21] Singer S, Ziegler C, Schwalenberg T, Hinz A, Gotze H, Schulte T. Quality of life in patients with muscle invasive and non-muscle invasive bladder cancer. Support Care Cancer. 2013;21(5):1383-93.

[22] Fung C, Pandya C, Guancial E, Noyes K, Sahasrabudhe DM, Messing EM, et al. Impact of bladder cancer on health 
related quality of life in 1,476 older Americans: a crosssectional study. J Urol. 2014;192(3):690-5.

[23] Schmidt S, Frances A, Lorente Garin JA, Juanpere N, Lloreta Trull J, Bonfill X, et al. Quality of life in patients with non-muscle-invasive bladder cancer: one-year results of a multicentre prospective cohort study. Urol Oncol. 2015;33(1):19 e7-e5.

[24] Cox E, Saramago P, Kelly J, Porta N, Hall E, Tan WS, et al. Effects of Bladder Cancer on UK Healthcare Costs and Patient Health-Related Quality of Life: Evidence From the BOXIT Trial. Clinical Genitourinary Cancer. 2020;18(4):e418-e42.

[25] Erkilic E, Kesimci E, Soykut C, Doger C, Gumus T, Kanbak O. Factors associated with preoperative anxiety levels of Turkish surgical patients: from a single center in Ankara. Patient Prefer Adherence. 2017;11:291-6.

[26] Jadoon NA, Munir W, Shahzad MA, Choudhry ZS. Assessment of depression and anxiety in adult cancer outpatients: a cross-sectional study. BMC Cancer. 2010;10:594.
[27] Kumar A, Dubey PK, Ranjan A. Assessment of Anxiety in Surgical Patients: An Observational Study. Anesth Essays Res. 2019;13(3):503-8.

[28] Mitchell AJ, Chan M, Bhatti H, Halton M, Grassi L, Johansen C, et al. Prevalence of depression, anxiety, and adjustment disorder in oncological, haematological, and palliative-care settings: a meta-analysis of 94 interviewbased studies. Lancet Oncol. 2011;12(2):160-74.

[29] Vartolomei L, Ferro M, Mirone V, Shariat SF, Vartolomei MD. Systematic Review: Depression and Anxiety Prevalence in Bladder Cancer Patients. Bladder Cancer. 2018;4(3):319-26.

[30] Jung A, Nielsen ME, Smith SK, Crandell J, Palmer $\mathrm{MH}$, Bryant AL, et al. Uncertainty, post-traumatic stress, and quality of life in non-muscle-invasive bladder cancer survivors. Journal of Clinical Oncology. 2017; 35(15_suppl):e16027-e. 